\title{
Omalizumab Treatment for Prevention of Anaphylaxis
}

\author{
Hector Rodriguez, MD \\ John Fahrenholz, MD*
}

\author{
Address \\ *Vanderbilt Asthma, Sinus, and Allergy Program Clinic, 2611 West End Avenue, \\ suite 120, Nashville, TN 37203, USA \\ Email: john.m.fahrenholz@vanderbilt.edu
}

Published online: 20 June 2014

(C) Springer International Publishing AG (outside the USA) 2014

Keywords Anaphylaxis - Urticaria - Idiopathic · Allergy · Hypersensitivity · Omalizumab

\section{Opinion Statement}

RCTs utilizing a specific treatment followed by meta-analysis of multiple RCTs for the specific treatment are the cornerstone of evidence-based medicine. This approach provides key insights into the average response of a therapeutic agent in the selected patient population for the target disease. Generally speaking, a developing trend is more investigations of medical therapies aiming to describe individual patient variability; further, such investigations typically aim to discover and subsequently understand this variability based on biomarkers or even disease-specific genetic polymorphisms among patients. This research returns us to the examination room where we treat individual patients. Given the complexity of idiopathic anaphylaxis and the currently available literature, we believe the time is now to initiate treatment trials of omalizumab in patients not well controlled with prior standard therapy. The use of prolonged systemic steroids should be avoided in favor of a trial of omalizumab. We do not intend to minimize the importance of double-blind placebo-controlled trials. However, based on the full spectrum of current evidence, we do not want to be blinded at the present time by the absence of a published RCT specific to idiopathic anaphylaxis.

\section{Introduction}

Omalizumab is a humanized IgG1 monoclonal antibody that binds selectively to the heavy chain constant region (FC) of human IgE. More specifically, omalizumab binds to human IgE within the $\mathrm{CH} 3$ region of the Fc portion of IgE. The $\mathrm{CH} 3$ portion of human IgE binds physiologically to the high-affinity IgE receptor (Fc $\in R I)$ on mast cells and basophils; IgE binding to Fc $€ \mathrm{RI}$ receptors along with IgE-IgE crosslinking triggers the- se effector cells to activate and release inflammatory mediators that can lead to anaphylaxis. The binding of omalizumab to the $\mathrm{CH} 3$ portion of human IgE essentially blocks the binding of omalizumab-human IgE complexes to Fc $€$ RI receptors on mast cells and basophils; thus, omalizumab-human IgE complexes cannot activate mast cells or basophils. Omalizumab therapy leads to a rapid decrease in total free serum IgE as omalizumab-human 
IgE complexes are cleared from the circulation through the reticuloendothelial system. In the setting of decreased free total IgE, high affinity IgE receptors on basophils and mast cells are downregulated. This provides a physiologic explanation for the decrease in basophil and mast cell activation that has been documented in patients receiving treatment with omalizumab therapy [1,2]. Given the key roles of mast cell activation and/or basophil activation in the pathophysiology of IgE-mediated anaphylaxis, including activation-induced release of key mediators such as histamine, leukotrienes and prostaglandins, investigators hypothesized that treating patients with omalizumab had the potential to prevent and/or reduce the severity of anaphylactic reactions in susceptible individuals [3].

Although neither a precise incidence nor lifetime prevalence of anaphylaxis has been definitively established, an expert consensus working group estimated the lifetime prevalence to be $1 \%$. However, this panel further concluded that the incidence of anaphylaxis was clearly increasing even though many cases are likely not reported [4]. The most commonly identified triggers of antigen-specific IgE-antibody mediated anaphylaxis are foods, medications and hymenoptera insect stings [3]. Subcutaneous allergen immunotherapy is used most commonly for the treatment of medication refractory allergic rhinoconjunctivitis as well as in patients who have experienced anaphylaxis caused by hymenoptera insect stings. Treatment of the latter group of patients decreases the recurrence rate of anaphylaxis related to subsequent hymenoptera stings by $90 \%$ or more in most patients [5]. While relatively uncommon, patients undergoing treatment with subcutaneous allergen immunotherapy for allergic rhinitis or stinging insect allergy do experience systemic allergic reactions. Most such systemic reactions are mild, involving cutaneous manifestations such as urticaria and pruritis with or without a simultaneous flare of rhinitis and/or conjunctivitis. Nevertheless, rare potentially life-threatening and even fatal anaphylaxis can occur [5].

Even after careful evaluation, no external trigger is identified in many patients who experience recurrent episodes of anaphylaxis $[6 \bullet]$. After ruling out other potential causative or complicating disorders, such as systemic mastocytosis, the diagnosis of idiopathic anaphylaxis can be made as a diagnosis of reasonable exclusion. The critical importance of ongoing questioning and searching for the possibility of previously unidentified trigger(s) is illustrated by the relatively recent description of hypersensitivity reactions including anaphylaxis related to IgE antibodies specific for the oligosaccharide galactose- $\alpha$-1,3-galactose (alpha-gal) [7]. Ingestion of mammalian meat including but not limited to beef, pork and lamb, which contain the alpha-gal carbohydrate, has been implicated as a causative factor in cases of anaphylaxis with onset of symptoms approximately 3 to $6 \mathrm{~h}$ after ingestion of the inciting mammalian meat trigger [7]. This type of delayed IgE-mediated reaction to any food had not previously been described. Given this key discovery, many cases of anaphylaxis that may have been labeled "idiopathic" in the past are now diagnosed as alpha-gal allergy. Alpha-gal is reviewed in detail in a separate review within this series.

Idiopathic anaphylaxis is not rare, but similar to all cause anaphylaxis, the exact incidence is not known. A practical "real world" perspective involving adults reviewed 601 patients who were evaluated for anaphylaxis of unknown cause at a single allergy practice with special interest and expertise in the diagnosis and management of anaphylaxis. Patients who were diagnosed with stinging insect anaphylaxis as well as those who experienced systemic reactions caused by subcutaneous allergen immunotherapy were excluded. A total of 356 of the 601 patients (59\%) were reported to have idiopathic anaphylaxis [8]. In children, the estimated incidence of idiopathic anaphylaxis is much lower at approximately $10 \%[9,10]$. Fortunately, even though the syndrome of idiopathic anaphylaxis in adults is relatively common, fatal anaphylaxis related to this condition remains a rare occurrence with only three fatalities reported in the literature to our knowledge $[11,12]$. Nevertheless, the negative impact on an individual patient's quality of life can be profound.

\section{Treatment}

\section{Peanut allergy}

- In 2003, Leung and colleagues published the first randomized doubleblind placebo controlled trial (RCT) of "omalizumab-like" therapy 
targeted at preventing or decreasing allergic reactions in patients with confirmed peanut allergy [13]. They used the monoclonal antibody, TNX-901. Patients received the study drug or placebo every 4 weeks for a total of four doses. Peanut allergy was confirmed by double-blind placebo-controlled graded oral peanut challenge (DBPCPC) prior to the study. Following the fourth dose of TNX-901 or placebo, the patients (total of 84 randomized to placebo or anti-IgE therapy) repeated the double-blind graded oral peanut protein challenge. Compared to baseline oral challenge, patients who received the highest of the three doses, $450 \mathrm{mg}$, showed a significantly increased ability to tolerate peanut compared to placebo. The baseline threshold dose of peanut protein causing mild to moderate anaphylaxis was approximately half a peanut $(178 \mathrm{mg})$. Following treatment, the threshold dose in the high dose arm approached the equivalent of nine peanuts $(2,805 \mathrm{mg})$. However, $25 \%$ of the patients in the high dose group did not show an increase in threshold peanut dose. Thus, while the results were overall positive, the individual variability in response was an important finding that was unexplained. This early study was not designed to include physiologic markers that could have potentially provided some insight in this regard. Overall, the study suggested that anti-IgE therapy had the potential to protect most peanut allergic patients from potentially fatal reactions related to accidental peanut ingestion.

- In a subsequent study using omalizumab, the same group of investigators aimed to confirm the positive findings of the prior peanut allergy study utilizing a similar study design but in a larger patient population. The investigators performed graded oral peanut challenge utilizing essentially the same (DBPCPC) as in the prior 2003 study. The target number for randomization was 150 participants. Unfortunately, two participants experienced severe anaphylactic reactions during the baseline peanut challenge portion of the study prior to the randomization phase. These two serious adverse events led to the study being stopped early based on the recommendation of the Data Safety Monitoring Committee. At the time of study termination, only 14 patients had completed repeat blinded graded peanut challenge following 24 weeks of omalizumab therapy at a total dose of $0.016 \mathrm{mg} / \mathrm{kg} / \mathrm{IgE}(\mathrm{IU} / \mathrm{ml})$ monthly. Among the $14 \mathrm{pa}-$ tients who had completed the study, nine had been receiving treatment with omalizumab and five had been receiving placebo. While trends in increased threshold doses for peanut-induced allergic reactions were seen, the study was not able to confirm findings from the previous study [14].

- In 2012, the results of an open-label trial of omalizumab in adult peanut allergic patients looked again for a protective effect as measured by response to DBPCPC. The investigators also measured the effects of treatment on effector cells, principally basophils. Of the 14 participants, 10 were able to complete all three blinded peanut challenges. The ten patients who completed the study showed a median 56-fold increase in peanut oral tolerance (baseline peanut 
protein threshold dose $=80 \mathrm{mg}$, omalizumab treatment phase median peanut protein threshold dose $=6,500 \mathrm{mg}$; one peanut $\approx 300 \mathrm{mg}$ protein). Peanut-induced basophil histamine release was completely suppressed in five patients, while in the nine others a ten-fold increase in peanut dose was required to induce the prior maximal histamine release response. The first food challenge while receiving omalizumab treatment took place 2-8 weeks after treatment initiation based on the study design, which called for this challenge to occur prior to week 8 if basophil histamine response had decreased to less than $20 \%$ of baseline $(n=5)$. The final peanut oral challenge was given at week 24 .

Even with the limitation of an open-label design, this investigation provided details that contributed valuable mechanistic insights and again demonstrated individual patient variability in treatment response; this variability was not explained by differences in basophil histamine response alone. At the time of the second oral peanut challenge, two patients showed little to no change in peanut threshold dose; these two patients did show an increased threshold dose approaching 2,000 mg during the oral peanut challenge at week 24 . Three other individuals reacted at the threshold dose of approximately $2,000 \mathrm{mg}$ at week 24 . In contrast, six patients had an increased threshold dose of peanut protein of approximately $10,000 \mathrm{mg}$ or greater during the second oral peanut challenge. Two of these patients did not complete the final oral peanut challenge. The four of these six patients who did complete the study maintained this robust increase in peanut protein threshold dose at week 24 challenge. With open-label treatment, all peanut allergic patients in this study tolerated at least the equivalent of $\approx$ six peanuts during at least one of the double-blind oral peanut challenges [15].

\section{Subcutaneous allergen immunotherapy}

- Casale and colleagues investigated the use of omalizumab for prevention of anticipated systemic allergic reactions observed in prior reports with the use of a rapid desensitization (RUSH) protocol for subcutaneous ragweed immunotherapy in adults. A total of 159 patients with ragweed allergic rhinitis in this RCT received either two total monthly doses of omalizumab $[0.016 \mathrm{mg} / \mathrm{kg} / \mathrm{IgE}(\mathrm{IU} / \mathrm{ml})]$ or placebo. This treatment was followed by single-day RUSH ragweed subcutaneous immunotherapy (to a dose of 1.2-4.0 $\mu \mathrm{g}$ of the major short ragweed protein allergen, Amb a 1) or placebo subcutaneous injections. Subsequently, patients continued every 2- or 4-week treatment with omalizumab versus placebo along with ragweed immunotherapy versus placebo immunotherapy for an additional 12 weeks. Among patients receiving active ragweed immunotherapy, those who were also receiving omalizumab demonstrated a significantly decreased risk of anaphylaxis caused by ragweed RUSH immunotherapy (2.6\% versus $15 \%$ of patients). The 
authors concluded that omalizumab pretreatment significantly improved the safety of ragweed RUSH immunotherapy [16].

- A RCT performed by Massanari and colleagues [17••] investigated the utility of adjunctive omalizumab therapy in patients with persistent allergic asthma with allergy triggers identified to cat, dog and/ or dust mite. The inclusion criteria required the patients to have ongoing asthma symptoms that were not well controlled with standard inhaled corticosteroid therapy. The patients continued inhaled corticosteroid therapy, and a total of 275 such patients were randomized to receive omalizumab or placebo for 16 weeks. At week 13, all patients initiated subcutaneous allergen immunotherapy, which included standard target allergen doses of at least one of the above allergens (two or all three based on individual skin test results) given using a cluster dose escalation protocol. The cluster portion of immunotherapy essentially overlapped the double-blind omalizumab or placebo-dosing period. Patients continued stable maintenance doses of immunotherapy for an additional 7 weeks. Omalizumab-treated patients had significantly decreased systemic allergic reactions to allergen immunotherapy compared to placebotreated patients (13.5\% versus $26.2 \%$ ). Further, patients receiving omalizumab treatment demonstrated improved asthma symptoms and were more likely to achieve the target allergen immunotherapy dose compared to placebo.

- Several case reports have suggested omalizumab therapy may prevent subsequent episodes of anaphylaxis caused by stinging insect immunotherapy injections. These reports include patients with and without underlying systemic mastocytosis. Several of these case reports suggest variability in effective dose as well as time to treatment response [18-22]. No RCT data have been published to date, but the case reports taken together are certainly suggestive of "real" benefit. In light of the efficacy demonstrated in the RCTs discussed above with RUSH IT using ragweed allergen mixtures and cluster IT using dust mite, cat and/or dog allergen mixtures, a trial of omalizumab therapy aiming to prevent or reduce reactions caused by stinging insect IT therapy should be attempted in select at-risk patients in our opinion. As mentioned in the introduction, stinging insect IT has proven efficacy in protecting patients from potentially fatal future insect sting reactions [5].

- No RCT has been published evaluating omalizumab treatment for the prevention of anaphylaxis in patients diagnosed with idiopathic anaphylaxis. However, investigators at the National Institute of Health (NIH) are actively recruiting patients for a single-center RCT of omalizumab therapy in this patient population (ClinicalTrials.gov 
Identifier: NCT00890162). At the time of preparation of this review, a total of 14 patients had been randomized to omalizumab or placebo. Twelve patients have completed the trial (personal communication with principal investigator, Melody Carter, M.D., at the National Institute of Allergy and Infectious Disease).

Even though no RCTs have been published specifically addressing idiopathic anaphylaxis, important insight into this potential therapeutic use comes from studies in patients with chronic idiopathic urticaria. A pivotal phase III RCT published in 2013 included 323 patients with chronic idiopathic urticaria refractory to at least "high dose" antihistamine therapy (typically four times the approved $\mathrm{H} 1$ antihistamine dose for the treatment of allergic rhinitis). Omalizumab doses of $150 \mathrm{mg}$ every 4 weeks and $300 \mathrm{mg}$ every 4 weeks demonstrated statistically significant improvement in both the primary and secondary endpoints, which included patient-reported standard symptom scores of pruritis severity and the total number/maximum size of urticarial lesions [23]. Given that mast cell and basophil activation are important in the pathogenesis of both disease processes and approximately $90 \%$ of patients who experience an anaphylactic reaction have skin manifestations including urticaria, this study in our opinion suggests that many patients with idiopathic anaphylaxis are likely to benefit from omalizumab treatment.

- Presently, publications of omalizumab therapy in the setting of idiopathic anaphylaxis are limited to case reports and small case series. In 2008, Jones and colleagues were the first to publish a case report of omalizumab therapy in a 48-year-old male who reported more than a decade of recurrent idiopathic anaphylaxis despite prophylactic treatment including antihistamines, antileukotrienes and systemic corticosteroids [24]. The frequency of his anaphylactic episodes without daily corticosteroid therapy occurred at a rate of at least six episodes yearly. With daily systemic corticosteroid therapy, the frequency and severity of episodes decreased but continued to occur at least four times in the year prior to initiation of omalizumab. His specific anaphylactic symptoms involved the skin with urticaria and flush; respiratory with wheezing and chest tightness; gastrointestinal with cramping pain and diarrhea; cardiovascular with hypotension. Urinary histamine metabolite elevation was documented during multiple anaphylactic episodes but remained normal at baseline. Bone marrow biopsy, which had been performed "multiple" times, was reported as normal; specifically, the bone marrow specimen obtained at our institution did not reveal abnormal numbers or morphology of mast cells and was negative for C-KIT D816V mutation. After initiation of omalizumab at a dose of $375 \mathrm{mg}$ every 2 weeks, he had no further episodes of anaphylaxis during the course of a year and was able to discontinue oral corticosteroids within this time frame as well. The authors' most recent 
contact with this patient was in 2013; he remained on omalizumab therapy and reported sustained control with no anaphylactic reactions requiring epinephrine and/or acute emergency care. He continued to take oral $\mathrm{H} 1$ and $\mathrm{H} 2$ blocking antihistamines; he reported periodic mild symptom flares involving central flush with or without urticaria (personal communication). Since that time multiple case reports have appeared in the world literature describing complete or partial control of idiopathic anaphylaxis previously refractory to standard therapies [25-27]. Many reports, particularly in patients with underlying systemic mastocytosis or monoclonal mast cell activation syndrome, have reported a longer period of time before onset of symptomatic improvement. Further, select patients have required more frequent dosing and/or a need for higher doses of therapy [28, 29]. In a case series of four patients with monoclonal mast cell activation or systemic mastocytosis, three experienced significant improvement, but in two of the patients (a male with aggressive systemic mastocytosis and a female with monoclonal mast cell activation syndrome), improvement was not clearly evident until they had been receiving omalizumab therapy for 6 months. One of the patients did not tolerate omalizumab related to adverse effects including headache, nausea and dizziness, which were more severe following a second dose of omalizumab. These symptoms were attributed to mast cell mediator release presumably based on her clinical history; objective measures of mediator release were not reported in this individual. However, in two of the patients who responded to therapy, serum tryptase levels and/or urine histamine metabolite decreased along with the need for oral corticosteroids [30]. The findings of decreasing serum tryptase and/or decreasing levels of histamine urinary metabolite in association with clinical improvement with omalizumab therapy were not observed in the previously cited case reports in other patients with systemic mastocytosis [28, 29].

\title{
Class of drugs (monoclonal antibody)
}

\section{Omalizumab}

\author{
Standard dosage \\ $150 \mathrm{mg}$ or $300 \mathrm{mg}$ subcutaneous injection every 4 weeks for chronic idiopathic \\ urticaria \\ Contraindications prior hypersensitivity reaction \\ Main side effects \\ local injection site reactions; anaphylaxis $0.2 \%$ \\ Special points Further study needed to determine optimal dosing in chronic idiopathic \\ urticaria; initial randomized controlled trial underway in patients with \\ idiopathic anaphylaxis
}

Cost/cost-effectiveness Wholesale acquisition cost in 2014, \$820/150 mg vial; expensive 


\section{Pediatric considerations}

- Multiple clinical investigations have evaluated the efficacy and safety of omalizumab use in children for a variety of allergic conditions.

RCTs of omalizumab performed in children with persistent asthma ages 6 through 11 have demonstrated efficacy and relative safety (not FDA approved) [31•]. FDA approved for allergic asthma ages 12 years and older. FDA approved for chronic idiopathic urticaria ages 12 years and older [32].

\section{Compliance with Ethics Guidelines}

Conflict of Interest

Hector Rodriguez and John Fahrenholz declare that they have no conflict of interest.

Human and Animal Rights and Informed Consent

This article does not contain any studies with human or animal subjects performed by any of the authors.

\section{References and Recommended Reading}

Papers of particular interest, published recently, have been highlighted as:

- $\quad$ Of importance

- Of major importance

1. Chanez P et al. Omalizumab-induced decrease of Fc $€$ RI expression in patients with severe allergic asthma. Resp Med. 2010;104:1608-17.

2. Beck L et al. Omalizumab-induced reductions in mast cell Fc €RI expression and function. J Allergy Clin Immunol. 2004;114:528-30.

3. Adkinson NF, Bochner BS, et al. Anaphylaxis. In: Middleton's allergy: principles and practice. Eighth Edition. 77, 1237-59. Copyright ${ }^{\odot} 2014$ by Saunders, an imprint of Elsevier Inc.

4. Lieberman P, Camargo Jr C, Bohlke K, et al. Epidemiology of anaphylaxis: findings of the American College of Allergy, Asthma and Immunology Epidemiology of Anaphylaxis Working Group. Ann Allergy Asthma Immunol. 2006;97:596-602.

5. Cox L, Nelson H, Lockey R. Allergen immunotherapy: a practice parameter third update. J Allergy Clin Immunol. 2011;127:S1-55.

6. $\quad$ Greenberger P, Lieberman P. Idiopathic anaphylaxis. J Allergy Clin Immunol Pract. 2014;2:243-50.

This article provides a balanced comprehensive clinically focused review. The authors include a historical description with reference to the initial description of the syndrome along with key elements of the evolution of the literature including current theories of pathogenesis as well as a state-of-the-art approach to diagnosis and treatment.

7. Commins S, Platts-Mills T. Anaphylaxis syndromes related to a new mammalian cross-reactive carbohydrate determinant. J Allergy Clin Immunol. 2009;124:652-7.

8. Webb L, Lieberman P. Anaphylaxis: a review of 601 cases. Ann Allergy Asthma Immunol. 2006;97:39-43.

9. Hogan MB, Kelly MA, Wilson NW, et al. Idiopathic anaphylaxis in children. Ann Allergy Asthma Immunol. 1998;81:140-2.

10. Patterson R, Harris KE. Idiopathic anaphylaxis. Allergy Asthma Proc. 1999;20:311-5.

11. Krasnick J, Patterson R, Meyers GL. A fatality from idiopathic anaphylaxis. Ann Allergy Asthma Immunol. 1996;76:376-8.

12. Patterson R, Clayton DE, Booth BH, et al. Fatal and near fatal idiopathic anaphylaxis. All Asthma Proc. 1995; 16:103-8.

13. Leung D, Sampson HA, Yunginger JW, et al. Effect of Anti-IgE therapy in patients with peanut allergy. N Engl J Med. 2003;348:986-93. 
14. Sampson HA, Leung D, Burks AW, et al. A phase II, randomized, double-blind, parallel-group, placebocontrolled oral food challenge trial of Xolair (omalizumab) in peanut allergy. J Allergy Clin Immunol. 2011;127:1309-10.

15. Savage JH, Courneya JP, Sterba MS, et al. Kinetics of mast cell, basophil, and oral food challenge responses in omalizumab-treated adults with peanut allergy. J Allergy Clin Immunol. 2012;130:1123-9.

16. Casale TB, Busse WW, Kline JN, et al. Omalizumab pretreatment decreases acute reactions after rush immunotherapy for ragweed-induced seasonal allergic rhinitis. J Allergy Clin Immunol. 2006;117:134-40.

17.• Massanari M, Nelson H, Casale T, et al. Effect of pretreatment with omalizumab on the tolerability of specific immunotherapy in allergic asthma. J Allergy Clin Immunol. 2010;125:383-9.

This randomized controlled trial provides solid evidence that adjunctive treatment with omalizumab in patients with allergic asthma not controlled on standard therapy including moderate to high dose ICS can show improvement in asthma control and more safely provide the addition of allergen immunotherapy.

18. Kontou-Fili K. High omalizumab dose controls recurrent reactions to venom immunotherapy in indolent systemic mastocytosis. Allergy. 2008;63:376-8.

19. Kontou-Fili K, Fili CI. Prolonged high-dose omalizumab is required to control reactions to venom immunotherapy in mastocytosis. Allergy. 2009;64:1384-5.

20. Kontou-Fili $\mathrm{K}$ et al. Omalizumab monotherapy for bee sting and unprovoked "anaphylaxis" in a patient with systemic mastocytosis and undetectable specific IgE. Ann Allergy Asthma Immunol. 2010;104:537-9.

21. Galera C, Soohun N, Zanker N, et al. Severe anaphylaxis to bee venom immunotherapy: efficacy of pretreatment and concurrent treatment with omalizumab. J Investig Allergol Clin Immunol. 2009;19:225-9.
22. Tartibi HM et al. Successful use of omalizumab for prevention of fire ant anaphylaxis. J Allergy Clin Immunol. 2010;126:664-5.

23. Maurer M, Rosen $\mathrm{K}, \mathrm{Hsin}-\mathrm{Ju} \mathrm{H}$, et al. Omalizumab for the treatment of chronic idiopathic or spontaneous urticaria. N Engl J Med. 2013;368:924-35.

24. Jones JD, Marney SM, Fahrenholz JM. Idiopathic anaphylaxis successful treated with omalizumab. Ann Allergy Asthma Immunol. 2008;101:550-1.

25. Warrier P, Casale TB. Omalizumab in idiopathic anaphylaxis. Ann Allergy Asthma Immunol. 2009; 102:257-8.

26. Demirturk M, Gelincik A, Colakoglu B, et al. Promising option in the prevention of idiopathic anaphylaxis: omalizumab. J Dermatol. 2012;39:552-4.

27. Bell M, Jackson D. Prevention of anaphylaxis related to mast cell activation syndrome with omalizumab. Ann Allergy Asthma Immunol. 2012;108:383-4.

28. Carter MC, Robyn JA, Bressler PB, et al. Omalizumab for the treatment of unprovoked anaphylaxis in patients with systemic mastocytosis. J Allergy Clin Immunol. 2007;119:1550-1.

29. Douglass JA, Carroll K, Voskamp A, et al. Omalizumab is effective in treating systemic mastocytosis in a nonatopic patient. Allergy. 2010;65:926-7.

30. Molderings GJ, Raithel M, Kratz F, et al. Omalizumab treatment of systemic mast cell activation disease: experiences from four cases. Intern Med. 2011;50:611-5.

31. Baena-Cagnani CE, Gómez RM. Current status of therapy with omalizumab in children. Curr Opin Allergy Clin Immunol. 2014;14:149-54.

The article reviews currently available clinical trial data regarding the safety and efficacy of omalizumab in pediatric patients. Further, use in evolving areas such as food allergy with oral immunotherapy and anaphylaxis is discussed.

32. http://www.accessdata.fda.gov/scripts/cder/ drugsatfda/index.cfm?fuseaction=Search.Label ApprovalHistory\#labelinfo. (Accessed June 2, 2014) 